\begin{tabular}{|l|l|l||}
\hline \multicolumn{2}{|c|}{ PublisherInfo } \\
\hline \hline PublisherName & $:$ & BioMed Central \\
\hline \hline PublisherLocation & $:$ & London \\
\hline \hline PublisherImprintName & $:$ & BioMed Central \\
\hline \hline
\end{tabular}

\title{
Mayo Clinic Site for Scleroderma
}

\begin{tabular}{|l|l|l||}
\hline \multicolumn{2}{|c|}{ ArticleInfo } \\
\hline \hline ArticleID & $:$ & 123 \\
\hline \hline ArticleDOI & $:$ & 10.1186 /ar-2001-3-webreport0018 \\
\hline \hline ArticleCitationID & $:$ & 0018 \\
\hline \hline ArticleSequenceNumber & $:$ & 80 \\
\hline \hline ArticleCategory & $:$ & Web Report \\
\hline \hline ArticleFirstPage & $:$ & 1 \\
\hline \hline ArticleLastPage & $:$ & 2 \\
\hline \hline & & RegistrationDate : 2001-1-17 \\
\hline ArticleHistory & $:$ & OnlineDate \\
\hline \hline ArticleCopyright & $:$ & Biomed Central Ltd2001-17 \\
\hline \hline ArticleGrants & $:$ & \\
\hline \hline ArticleContext & $:$ & 130753311 \\
\hline \hline
\end{tabular}




\section{Overview}

This site offers a summary description of scleroderma and its manifestations. This page, with its simple and clear statements, may be of interest to both physicians and their patients, as a means to disuss this disease. It also contains a patient-orientated link to the Scleroderma support initiative (http://www.sclerodermasupport.com/support/adaptation/index.htm offering online support and links to medical resources, as well as email and online chat groups.

\section{References}

1. MayoClinic.com. [http://www.mayohealth.org/home?id=HQ01334] 